\title{
Calcaneal quantitative ultrasound is associated with all-cause and cardiovascular disease mortality independent of hip bone mineral density
}

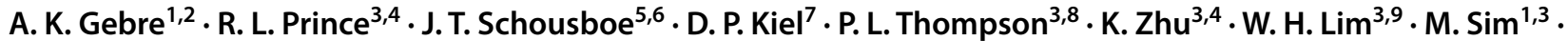 \\ J. R. Lewis ${ }^{1,3,10}$
}

Received: 26 May 2021 / Accepted: 20 January 2022 / Published online: 11 February 2022

(c) The Author(s) 2022

\begin{abstract}
Summary Osteoporosis has been linked with increased risk of cardiovascular disease previously. However, few studies have detailed bone and vascular information. In a prospective study of older women, we demonstrated heel quantitative ultrasound measures were associated with increased cardiovascular and all-cause mortality, independent of established cardiovascular risk factors.

Introduction Osteoporosis and low bone mineral density (BMD) have been previously linked to cardiovascular disease (CVD) and mortality. Calcaneal quantitative ultrasound (QUS) is used to evaluate bone material properties, especially in older women. However, it is uncertain whether it is related to risk of mortality. This study was aimed to investigate the association between calcaneal QUS measurements and 15-year all-cause and CVD mortality in 1404 older women (mean age $75.2 \pm 2.7$ years).

Methods One thousand four hundred four older women, participants of Calcium Intake Fracture Outcome study (CAIFOS), had calcaneal bone measured at baseline (1998) and followed for 15 years. The primary outcomes, any deaths, and deaths attributable to cardiovascular causes ascertained by using linked data were obtained from Western Australia data linkage system.

Results Over the 15 years of follow-up (17,955 person years), 584 of the women died, and 223 from CVD. For every standard deviation (SD), reduction in broadband ultrasound attenuation (BUA) in minimally and multivariable-adjusted model including cardiovascular risk factors increased relative hazards for all-cause (multivariable-adjusted HR 1.15; 95\%CI: $1.06-1.26, p=0.001$ ) and CVD mortality (multivariable-adjusted HR 1.20; 95\%CI: 1.04-1.38, $p=0.010$ ). Such relationships also persisted when hip BMD was included in the model (all-cause mortality HR 1.19; 95\%CI: 1.07-1.33, $p=0.002$; CVD mortality HR 1.28 ; $95 \%$ CI: $1.07-1.53, p=0.008)$.

Conclusion BUA is associated with all-cause and CVD mortality in older women independent of BMD and established CVD risk factors. Understanding why and how these are related may provide further insights about the bone-vascular nexus as well as therapeutic targets benefiting both systems.
\end{abstract}

Keywords All-cause mortality $\cdot$ Bone $\cdot$ Calcaneal quantitative ultrasound $\cdot$ Cardiovascular mortality

\section{Introduction}

Vascular and bone diseases are both chronic conditions associated with aging that cause considerable morbidity and mortality, and associated with substantial health and

J. R. Lewis

joshua.lewis@ecu.edu.au

Extended author information available on the last page of the article economic burden [1]. Shared genetic and lifestyle risk factors, chronic inflammation, impaired blood and nutrient supply, estrogen deficiency, oxidative stress, and vascular calcification may underlie the link between osteoporosis and cardiovascular disease (CVD) [2, 3]. Despite low bone mineral density (BMD) determined using dual-energy $\mathrm{x}$-ray absorptiometry (DXA) being linked to all-cause mortality [4], its relationship with other health outcomes apart from fracture remains unclear [5]. Vascular calcification, primarily of the abdominal aorta, has also been linked with higher 
risk of fracture in older people [6-8]. We and others have also identified an overlap in cytokine regulation of bone and vascular phenomena $[9,10]$. Specifically, an association between circulating vascular inflammatory biomarkers and bone health has been reported [11-13].

A number of studies and a recent meta-analysis of 10 prospective studies reported an association between lower hip and spine BMD with increased risk of CVD and allcause mortality [4]. A recent study also revealed an inverse relationship between speed of sound (SOS), measured using calcaneal quantitative ultrasound (QUS) and ischemic heart disease mortality [14]. However, only limited studies have assessed the association of other measures of bone material properties from QUS such as broadband ultrasound attenuation (BUA) and stiffness index (SI) and other skeletal sites with all-cause and CVD mortality. These parameters are thought to measure similar bone properties to DXA and have been demonstrated to predict fracture risk independently in older populations [15-17]. Presently, there are conflicting findings as to whether these measures of the material properties of bone predict all-cause or CVD mortality [18, 19].

Therefore, we sought to examine the association between baseline QUS measures with all-cause and CVD mortality over 15 years in a longitudinal cohort of older women. Additionally, we aimed to determine whether the associations of ultrasound bone parameters with all-cause and CVD mortality are modified by known CVD risk factors.

\section{Materials and methods}

The manuscript complies with the STROBE reporting guidelines for observational studies.

\section{Study population}

Older women aged over 70 years were recruited from the Western Australian general population of women by mail using the electoral roll in 1998 to a 5-year prospective, randomized, controlled trial of oral calcium supplements to prevent osteoporotic fractures, the Calcium Intake Fracture Outcome study (CAIFOS) [20]. Of the 5586 women approached, 1460 women were randomized to receive either; $1.2 \mathrm{~g}$ of elemental calcium as calcium carbonate daily $(n=730)$ or a matching placebo $(n=730)$ for 5 years as described previously [20]. At the conclusion of CAIFOS, participants were followed for a further 10 years of follow-up. Of the 1460 participants recruited, 1404 had calcaneal QUS measured at baseline (1998). Of these 705 participants were randomized to the calcium-treated group while 699 were randomized to the placebo group.

\section{Bone measurements}

QUS of the calcaneus of the left foot was obtained in duplicate using a Lunar Achilles Ultrasound machine (Lunar Corp., Madison, WI, USA) at baseline (1998) in 1404 participants. The average measurement of BUA, SOS, and SI were recorded. Coefficients of variation (CV) were $0.4 \%, 1.6 \%$, and $2.4 \%$ for SOS, BUA, and SI, respectively, in our laboratory [11].

Total hip areal bone mineral density was determined by DXA using the same Hologic Acclaim 4500A fan beam densitometer (Hologic Corp, Waltham, MA, USA) in a subset of 480 women at baseline and in 650 at year 1 of the intervention (total $n=1130$ ) due to a lack of financial resources at the time. The $\mathrm{CV}$ at the total hip was $1.2 \%$ in our laboratory [21].

\section{Baseline measures, risk factors, and asymptomatic CVD}

Baseline medical and medication history including the presence of diabetes were obtained from all participants. Participants' medical histories and medications were verified by their general practitioners where possible. Prevalent CVD was identified from hospital principal discharge diagnosis codes (ICD-9-CM codes 390-459) for the previous 18 years (1980-1998). Body mass index (BMI) was calculated in kilograms per square meter, weight $(\mathrm{kg})$ divided by height squared $\left(\mathrm{m}^{2}\right)$. Mean blood pressure was measured on the right arm with a mercury column manometer while the participants have been seated in an upright position. The level of physical activity was evaluated by a questionnaire [22, 23], with activity levels determined in kilocalories per day using a validated method utilizing body weight, questions on the number of hours, and type of physical activity including specific energy costs [24, 25]. Previous smoking history was considered smoking of at least one cigarette per day for at least 3 months at any time. Estimated 10-year cardiovascular risk was calculated using the Framingham Risk Scores (FRS) with either (a) non-laboratory-based variables: age, BMI, systolic blood pressure, antihypertensive medications, smoking status, and diabetes mellitus, or (b) the laboratory-based FRS, where high-density lipoprotein and total cholesterol replace BMI in the equations [26]. The presence of abdominal aortic calcification (AAC) was assessed on lateral single-energy images of the thoracolumbar spine taken in 1998/1999 using a Hologic 4500A machine (Hologic, Bedford, MA, USA) by single experienced investigator (JTS) as described previously [27]. AAC was classified into low $\mathrm{AAC}(\mathrm{AAC} 24$ score $=0$ and 1$)$ or moderate to severe AAC 
(AAC24 score $>1$ ). The presence of focal atherosclerotic carotid plaques was determined after 3 years (2001) using B mode carotid ultrasound examination by a single sonographer with an $8.0-\mathrm{mHz}$ linear array transducer fitted to an Acuson Sequoia 512 ultrasound machine using standard imaging as described previously [28].

\section{Biochemistry}

Fasting blood samples were collected at baseline (1998). Lipocalin 2, total cholesterol, high-density lipoprotein cholesterol, total L-homocysteine, and serum cystatin C were measured as previously described $[11,29]$.

\section{Mortality outcomes}

The primary outcome for this study was all-cause and CVD mortality. The outcomes were defined using primary cause of death coded death certificate data from the ICD 9 Clinical Modification (ICD-9-CM) [30], and the ICD, 10th Revision, Australian Modification (ICD-10-AM) [31]. Primary cause of death data was used for CVD death (codes included ICD-9-CM codes 390-459 and ICD-10-AM codes I00-I99); cancer death (codes included ICD-9-CM code 140-239 excluding 210-229 and ICD-10-AM code C00-D48 excluding D10-D36); and other deaths (all other codes). Primary cause of death diagnosis text field of the death certificate was used where coded data were not yet available to ascertain the cause of death. Three deaths were under coronial inquiry at the time of data extraction, and no primary cause of death was available.

\section{Statistical analysis}

QUS variables: BUA, SOS, and SI, were considered either continuous or quartiles in analyses with quartile 4 (highest bone quality) as the referent category. Pearson correlation coefficient was calculated for the correlation between QUS variables with age, BMI, and hip BMD. Baseline characteristics were stratified by 15 years of all-cause mortality (yes/no) and were presented as mean and standard deviation (SD) for continuous variables or as number and percentage for categorical variables. The covariates in the multivariable-adjusted Cox proportional hazards regression models included, model $1-$ minimally adjusted: age, BMI, and treatment code (calcium/placebo); model 2-multivariableadjusted: model 1 plus history of smoking, CVD, cancer, and diabetes; and model 3: model 2 plus hip BMD. Cox proportional hazards regression analysis was undertaken using SPSS (version 22; IBM Corporation, Armonk, NY) and STATA version 9.2 statistical software programs (Stata Corporation, College Station, TX). No violations of the Cox proportional hazards assumptions were detected. The dose-response relationship between calcaneal BUA and mortality outcomes were determined with restricted cubic splines using the R package "rms" [32] with $\mathrm{df}=3$ and using BUA at $100 \mathrm{db} / \mathrm{Mhz}$ as the reference level, adjusted for covariates as described in model 2 . For the analysis between BUA and all-cause and CVD mortality, $p<0.017$ (Bonferroni-corrected) in two-tailed testing was considered statistically significant. To determine whether there was an interaction between circulating inflammatory biomarkers, CVD risk factors, and subclinical measures of CVD with BUA for all-cause and CVD mortality, we adopted interaction testing using minimally adjusted model. $P_{\text {int }}<0.1$ was considered statistically significant. Moreover, we undertook further analyses after including additional covariates such as physical activity, 25OHD, and free estradiol index into the multivariable-adjusted model.

\section{Results}

\section{Baseline characteristics}

Baseline characteristics are presented in Table 1. Women that died over the 15 years of follow-up were older, more likely to have smoked in the past, and have a sedentary lifestyle, as well as present with prevalent diabetes or CVD at baseline. Correlation tests revealed that age was negatively correlated with measures of baseline calcaneal QUS: BUA $r=-0.13$, SOS $=-0.09$, and SI $r=-0.12$, all $p<0.001$, while BMI was positively correlated with BUA $r=0.34$, SOS $r=0.14$, and SI $r=0.25$, all $p<0.001$. Total hip BMD was also positively correlated with BUA $r=0.53$, SOS $r=0.49$, and SI $r=0.54$, all $p<0.001$.

\section{Calcaneal QUS measures and all-cause mortality}

Between baseline and year 1, there were 9 of the 1404 women $(0.6 \%)$ with BUA measurements that died. Each SD reduction in BUA, SOS, and SI was associated with an increased risk of all-cause mortality in the minimally and multivariable-adjusted models (Table 2). After adjusting for total hip BMD, calcaneal BUA and SI remained significantly related with a higher risk of all-cause mortality. However, the association between SOS and all-cause mortality was attenuated after adjustment for total hip BMD. Restricted cubic splines indicated a linear relationship between calcaneal BUA and all-cause mortality (Fig. 1a). Kaplan-Meier survival curves for the relationship between quartiles of BUA and all-cause mortality are shown in Fig. 2a. Percentages of deaths by quartiles of calcaneal BUA are shown in Fig. 3. Women with the lowest BUA (quartile 1) had 57\% and $58 \%$ greater relative hazard of all-cause mortality in the minimally and multivariable-adjusted models, respectively, 
Table 1 Baseline characteristics of the study population stratified by 15 -year mortality

\begin{tabular}{|c|c|c|c|c|}
\hline & All & Survivors & Any death & CVD death \\
\hline Number & 1404 & 820 & 584 & 223 \\
\hline Age, years & $75.2 \pm 2.7$ & $74.8 \pm 2.6$ & $75.7 \pm 2.8$ & $76.0 \pm 2.8$ \\
\hline Body mass index, $\mathrm{kg} / \mathrm{m}^{2}$ & $27.1 \pm 4.7$ & $26.9 \pm 4.3$ & $27.4 \pm 5.2$ & $27.6 \pm 5.3$ \\
\hline Calcium treatment, yes (\%) & $705(50.2)$ & $422(51.5)$ & $283(48.5)$ & $106(47.5)$ \\
\hline Smoked ever, yes (\%) & $514(36.8)$ & $278(34.1)$ & $236(40.5)$ & $80(36.0)$ \\
\hline Sedentary, yes $(\%)$ & $342(24.4)$ & $178(21.7)$ & $164(28.1)$ & $63(28.3)$ \\
\hline Physical activity, kcal/day & $112[25-204]$ & 122 [43-205] & $93(0-202)$ & $87[0-205]$ \\
\hline \multicolumn{5}{|l|}{ Prevalent disease } \\
\hline Diabetes, yes (\%) & $86(6.1)$ & $36(4.4)$ & $50(8.6)$ & $13(5.8)$ \\
\hline CVD, yes $(\%)$ & $331(23.6)$ & $153(18.7)$ & $178(30.5)$ & $80(35.9)$ \\
\hline Prevalent cancer, yes $(\%)$ & $72(5.1)$ & $34(4.1)$ & $38(6.5)$ & $7(3.1)$ \\
\hline \multicolumn{5}{|l|}{ Blood pressure $^{1}$} \\
\hline Systolic blood pressure, $\mathrm{mmHg}$ & $138 \pm 18$ & $138 \pm 18$ & $139 \pm 18$ & $140 \pm 19$ \\
\hline Diastolic blood pressure, $\mathrm{mmHg}$ & $73 \pm 11$ & $73 \pm 11$ & $74 \pm 12$ & $74 \pm 12$ \\
\hline Antihypertensive medications, yes (\%) & $605(43.1)$ & $317(38.7)$ & $288(49.3)$ & $122(54.7)$ \\
\hline Lipid lowering therapy, yes (\%) & $263(18.7)$ & $150(18.3)$ & $113(19.3)$ & $41(18.4)$ \\
\hline \multicolumn{5}{|l|}{ Lipid profiles ${ }^{2}$} \\
\hline Total cholesterol, $\mathrm{mmol} / \mathrm{L}$ & $5.9 \pm 1.1$ & $5.9 \pm 1.1$ & $5.8 \pm 1.2$ & $5.9 \pm 1.2$ \\
\hline $\mathrm{LDLC}, \mathrm{mmol} / \mathrm{L}$ & $3.7 \pm 1.0$ & $3.7 \pm 0.9$ & $3.6 \pm 1.1$ & $3.7 \pm 1.1$ \\
\hline $\mathrm{HDLC}, \mathrm{mmol} / \mathrm{L}$ & $1.5 \pm 0.4$ & $1.5 \pm 0.4$ & $1.4 \pm 0.4$ & $1.5 \pm 0.4$ \\
\hline Triglycerides, $\mathrm{mmol} / \mathrm{L}$ & $1.6 \pm 0.7$ & $1.5 \pm 0.7$ & $1.6 \pm 0.8$ & $1.5 \pm 0.7$ \\
\hline Framingham risk scores $(\mathrm{BMI})^{3}$ & $22.3 \%$ & $21.1 \%$ & $23.9 \%$ & $24.7 \%$ \\
\hline Framingham risk scores (lipids) ${ }^{4}$ & $19.0 \%$ & $18.1 \%$ & $20.6 \%$ & $21.1 \%$ \\
\hline \multicolumn{5}{|l|}{ Calcaneal quantitative ultrasound } \\
\hline BUA, db/Mhz & $100 \pm 8$ & $101 \pm 8$ & $100 \pm 8$ & $99 \pm 8$ \\
\hline Speed of sound, $\mathrm{m} / \mathrm{s}$ & $1513 \pm 26$ & $1515 \pm 25$ & $1510 \pm 26$ & $1510 \pm 25$ \\
\hline Stiffness index, \% & $71 \pm 11$ & $71 \pm 11$ & $69 \pm 11$ & $69 \pm 11$ \\
\hline Hip $\mathrm{aBMD}^{5}, \mathrm{~g} / \mathrm{cm}^{2}$ & $0.81 \pm 0.12$ & $0.82 \pm 0.12$ & $0.81 \pm 0.13$ & $0.80 \pm 0.12$ \\
\hline
\end{tabular}

Abbreviations: $C V D$, cardiovascular disease; $L D L C$, low-density lipoprotein cholesterol; $H D L C$, highdensity lipoprotein cholesterol; $B U A$, broadband ultrasound attenuation; $a B M D$, areal bone mineral density. Data expressed as mean \pm SD or number and (\%) or median and (IQR). Italicized numbers indicate a significant difference $(p \leq 0.05)$ from survivors by either univariate ANOVA with Tukey's post hoc test, Kruskal-Wallis $H$ or $\chi^{2}$ test for trend where appropriate

${ }^{1} n=1360$

${ }^{2} n=1001$

${ }^{3} n=1358$

${ }^{4} n=974$

${ }^{5} n=1130$

in comparison to those with the highest BUA (quartile 4); with this increasing to $67 \%$ after the inclusion of hip BMD, see Table 3. Noteworthy, hip BMD was not associated with all-cause (HR 0.42; 95\%CI: 0.08-2.17) and CVD (HR 0.75; 95\% CI: 0.28-1.97) mortality after accounting for BUA.

\section{Calcaneal QUS measures and CVD mortality}

Each SD reduction in BUA was consistently associated with an increased risk of CVD mortality in all models (Table 2). Specifically, each SD decrease in calcaneal BUA was associated with a $20 \%$ increase in the relative hazard for
CVD mortality in the multivariable-adjusted model, which increased to $28 \%$ after the inclusion of hip BMD in the multivariable-adjusted model (Table 2). Both SOS and SI were not associated with CVD mortality in the multivariableadjusted analysis. Restricted cubic splines indicated a linear relationship between calcaneal BUA and CVD mortality (Fig. 1b). Kaplan-Meier survival curves for the relationship between BUA and CVD mortality are presented in Fig. 2 b. Compared to individuals with the highest BUA (quartile 4), women with the lowest BUA (quartile 1) had a 59\% increased risk for CVD mortality in our multivariableadjusted analysis. The relative hazard slightly attenuated 
Table 2 Relative hazard per standard deviation (SD) reduction in calcaneal quantitative ultrasound measures

\begin{tabular}{|c|c|c|c|c|c|}
\hline & \multirow[t]{2}{*}{ SD } & \multicolumn{2}{|c|}{ All-cause mortality $(n=584)$} & \multicolumn{2}{|l|}{ CVD mortality $(n=223)$} \\
\hline & & Relative hazard (95\%CI) & $P$ value & Relative hazard (95\%CI) & $P$ value \\
\hline \multicolumn{6}{|l|}{ Model 1} \\
\hline BUA, db/Mhz & 8.0 & $1.15(1.05-1.25)$ & 0.002 & $1.21(1.05-1.38)$ & 0.008 \\
\hline $\mathrm{SOS}, \mathrm{m} / \mathrm{s}$ & 25.6 & $1.14(1.05-1.24)$ & 0.002 & $1.12(0.98-1.28)$ & 0.097 \\
\hline Stiffness index, $\%$ & 11.5 & $1.15(1.06-1.25)$ & 0.001 & $1.16(1.01-1.32)$ & 0.038 \\
\hline \multicolumn{6}{|l|}{ Model 2} \\
\hline BUA, db/Mhz & 8.0 & $1.15(1.06-1.26)$ & 0.001 & $1.20(1.04-1.38)$ & 0.010 \\
\hline $\mathrm{SOS}, \mathrm{m} / \mathrm{s}$ & 25.6 & $1.13(1.04-1.23)$ & 0.004 & $1.11(0.97-1.26)$ & 0.146 \\
\hline Stiffness index, \% & 11.5 & $1.15(1.06-1.25)$ & 0.001 & $1.14(1.00-1.31)$ & 0.057 \\
\hline \multicolumn{6}{|l|}{ Model $3^{\#}$} \\
\hline $\mathrm{BUA}, \mathrm{db} / \mathrm{Mhz}$ & 8.0 & $1.19(1.07-1.33)$ & 0.002 & $1.28(1.07-1.53)$ & 0.008 \\
\hline $\mathrm{SOS}, \mathrm{m} / \mathrm{s}$ & 25.6 & $1.09(0.98-1.22)$ & 0.115 & $1.07(0.90-1.29)$ & 0.440 \\
\hline Stiffness index, $\%$ & 11.5 & $1.15(1.03-1.28)$ & 0.017 & $1.16(0.96-1.39)$ & 0.126 \\
\hline
\end{tabular}

Abbreviations: $C V D$, cardiovascular disease; $B U A$, broadband ultrasound attenuation; $S D$, standard deviation; SOS, speed of sound. Model 1: age, BMI, and treatment (calcium/placebo). Model 2: model 1 plus smoking history, prevalent diabetes, CVD and cancer. Model 3: Model 2 plus total hip BMD in 1998/1999\# (assessed in 1130 participants). $P$ value represents from Cox proportional hazards regression with italicized Bonferroni-corrected $p$ values $\leq 0.017$ in the Bonferroni-corrected multivariable and hip BMDadjusted models (Table 3). No consistent associations were seen for cancer or "other" mortalities in unadjusted or adjusted models (data not shown). Moreover, prevalent CVD was associated with greater relative hazard for allcause (HR 1.53; 95\% CI: 1.28-1.82) and CVD mortality (HR 1.88; 95\% CI: 1.42-2.48) without accounting BUA. Prevalent CVD also remained significant after accounting for BUA (for

(a)

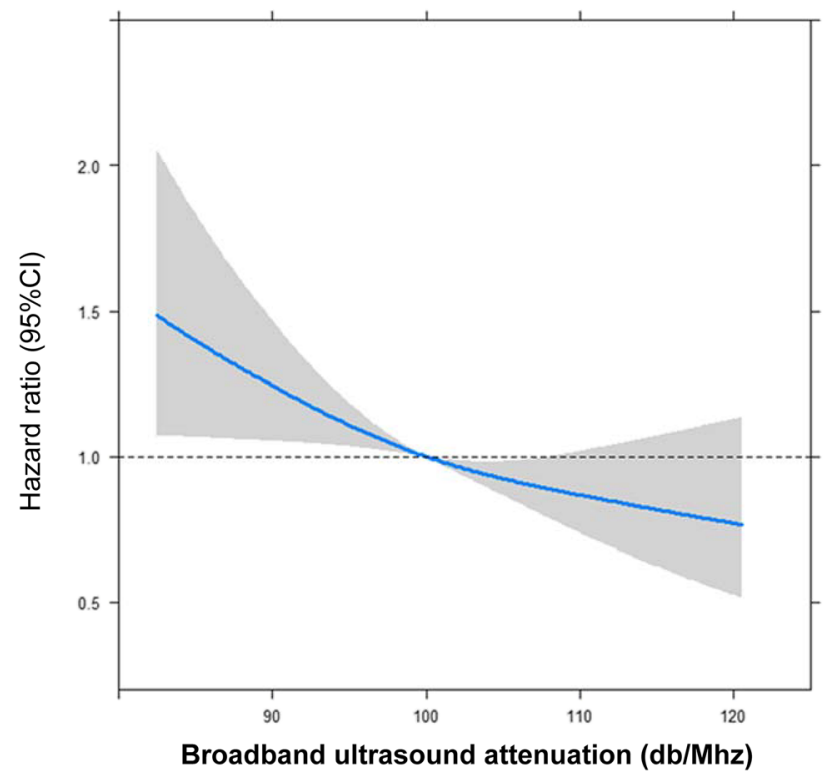

Fig. 1 Adjusted hazard ratios for calcaneal broadband ultrasound attenuation (BUA) in relation to risk of a all-cause mortality and $\mathbf{b}$ cardiovascular disease-related mortality based on fitted restricted cubic splines using $100 \mathrm{db} / \mathrm{Mhz}$ as the reference level. The multivar- all-cause HR 1.52; 95\%CI: $1.27-1.81$ and CVD mortality HR 1.86; 95\%CI: 1.41-2.45).

\section{Further analyses}

The association between BUA and all-cause and CVD mortality remained significant after excluding those without hip BMD measurement available at baseline

(b)

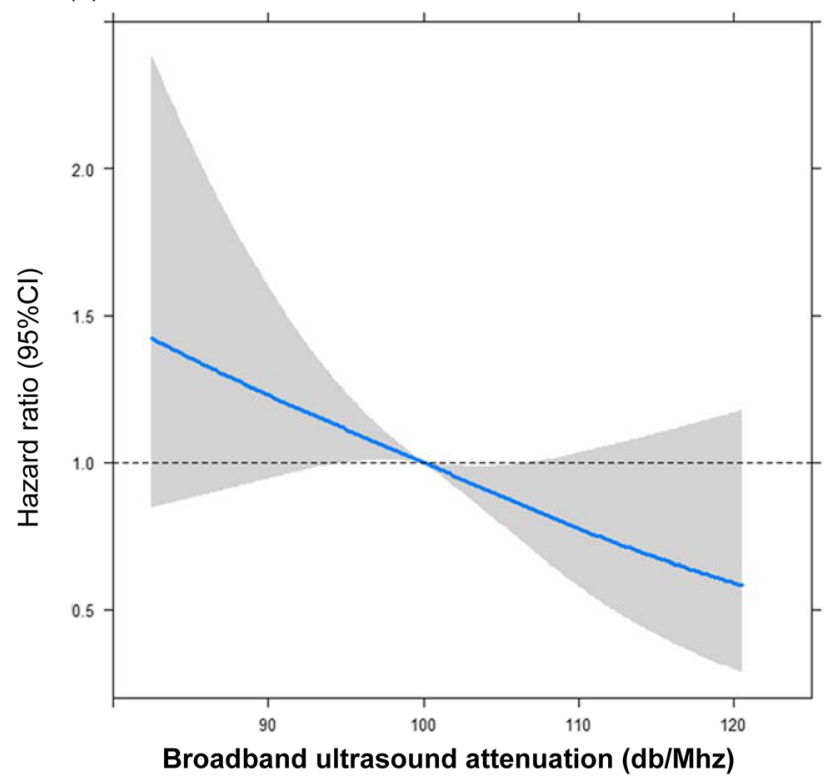

iable-adjusted model included age, BMI, treatment code (calcium/ placebo), smoked ever, history of CVD, history of cancer, and diabetes (model 2). Solid line is estimated HR and grey shading represent 95\% CI 


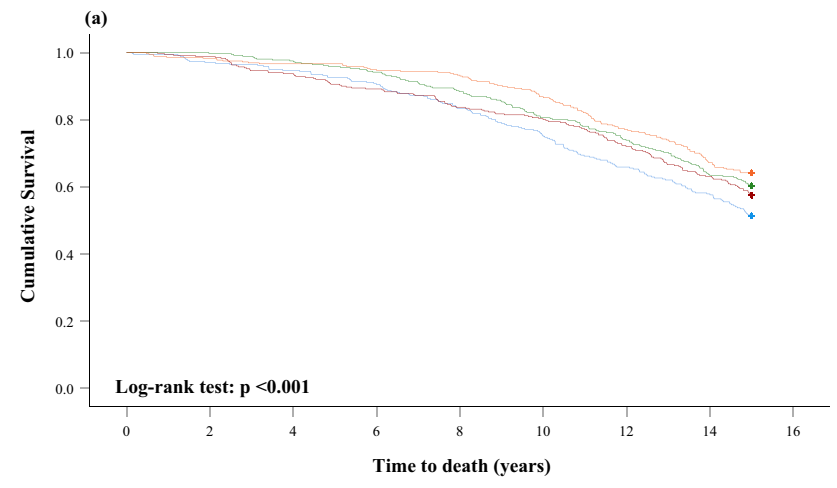

Fig. 2 Kaplan-Meier survival curves by quartiles of BUA a all-cause mortality $(n=584)$ and b CVD mortality $(n=223)$ over 15 years categorized by quartiles of calcaneal quantitative ultrasound broadband

in minimally and multivariable-adjusted models (Supplementary Table 1). It also remained significant after physical activity, total 25OHD, and free estradiol index were included into the hip BMD-adjusted model separately (Supplementary Table 2). We undertook further analyses adjusting for 10-year estimated cardiovascular risk using the non-laboratory- (BMI) and laboratory(total cholesterol and high-density lipoprotein cholesterol) calculated Framingham Risk Scores with treatment code and prevalent CVD and observed similar results (Supplementary Table 3). Given fractures have also been associated with increased risk of mortality, we undertook further analyses adjusting for prevalent fractures (per SD lower calcaneal BUA adjusted HR for all-cause mortality

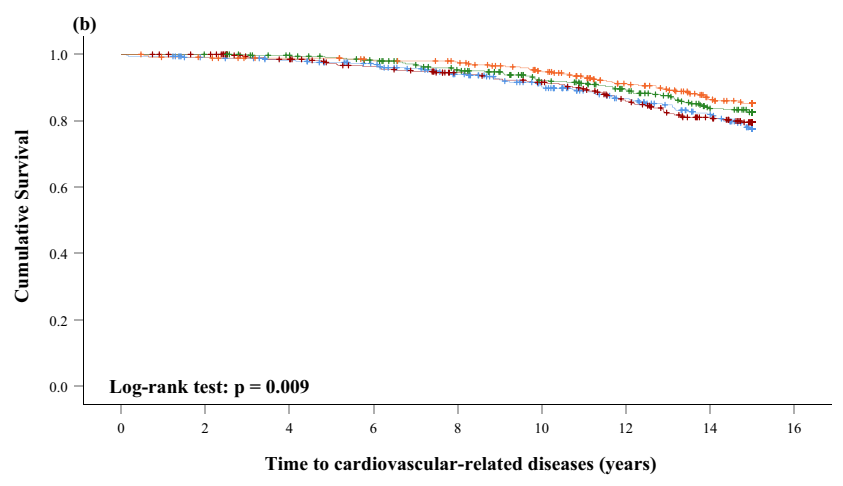

ultrasound attenuation (BUA). Quartiles 1, 2, 3, and 4 are represented by the blue, red, green, and orange lines, respectively

aHR 1.17; 95\%CI: $1.07-1.27, p=0.001$ and CVD mortality aHR 1.20; 95\%CI: $1.05-1.39, p=0.010$ ).

\section{Modifiers of the association between calcaneal BUA and all-cause and CVD mortalities}

Interaction testing between BUA and circulating vascular inflammatory biomarkers identified lipocalin 2, cystatin C, and homocysteine as potential modifiers of the association between BUA and all-cause and CVD mortalitiy $\left(P_{\text {int }}<0.05\right)$ (Fig. 4). Additionally, interactions between BMI and the presence of atherosclerotic carotid plaque with calcaneal BUA for all-cause mortality were noted. However, no such interactions were observed for CVD mortality (Supplementary Table 4).
Fig. 3 Percentage of deaths by quartiles of calcaneal BUA. Q1: 48.7\% died, Q2: $42.6 \%$ died, Q3: 39.7\% died, and Q4: 35.9\% died

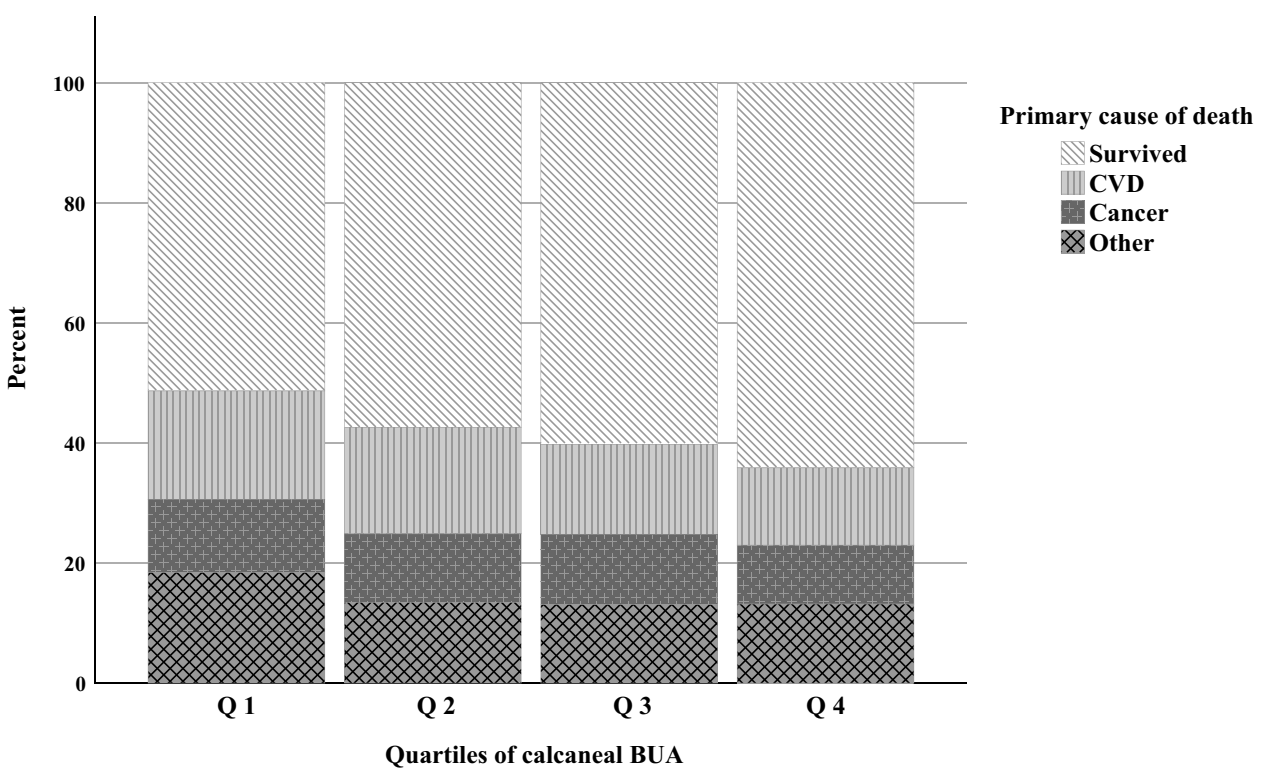


Table 3 Multivariable-adjusted hazard ratio for death by quartiles of calcaneal BUA

\begin{tabular}{|c|c|c|c|c|c|c|}
\hline & \multicolumn{3}{|l|}{ All deaths } & \multicolumn{3}{|c|}{ CVD deaths } \\
\hline & Number of deaths (\%) & Hazard ratio $(95 \% \mathrm{CI})$ & $P$ value & $\begin{array}{l}\text { Number of } \\
\text { deaths (\%) }\end{array}$ & Hazard ratio $(95 \% \mathrm{CI})$ & $P$ value \\
\hline \multicolumn{7}{|l|}{ Model 1} \\
\hline Quartile $1(n=337)$ & $164(48.7)$ & $1.57(1.23-2.00)$ & $<0.001$ & $61(18.1)$ & $1.63(1.09-2.44)$ & 0.016 \\
\hline Quartile $2(n=350)$ & $149(42.6)$ & $1.27(1.00-1.62)$ & 0.051 & $62(17.8)$ & $1.47(1.00-2.18)$ & 0.053 \\
\hline Quartile $3(n=360)$ & $143(39.7)$ & $1.20(0.91-1.58)$ & 0.204 & $54(15.0)$ & $1.28(0.86-1.90)$ & 0.220 \\
\hline Quartile $4(n=357)$ & $128(35.9)$ & 1.00 (referent) & & $46(13.0)$ & 1.00 (referent) & \\
\hline \multicolumn{7}{|l|}{ Model 2} \\
\hline Quartile $1(n=337)$ & $164(48.7)$ & $1.58(1.24-2.02)$ & $<0.001$ & $61(18.1)$ & $1.59(1.06-2.44)$ & 0.024 \\
\hline Quartile $2(n=350)$ & $149(42.6)$ & $1.28(1.01-1.64)$ & 0.045 & $62(17.8)$ & $1.51(1.02-2.24)$ & 0.041 \\
\hline Quartile $3(n=360)$ & 143 (39.7) & $1.21(0.95-1.54)$ & 0.120 & $54(15.0)$ & $1.29(0.86-1.92)$ & 0.214 \\
\hline Quartile $4(n=357)$ & $128(35.9)$ & 1.00 (referent) & & $46(13.0)$ & 1.00 (referent) & \\
\hline \multicolumn{7}{|l|}{ Model $3^{\#}$} \\
\hline Quartile $1(n=262)$ & $125(47.7)$ & $1.67(1.23-2.25)$ & 0.001 & $48(18.3)$ & $1.83(1.10-3.03)$ & 0.020 \\
\hline Quartile $2(n=275)$ & $111(40.4)$ & $1.30(0.98-1.74)$ & 0.073 & $46(16.7)$ & $1.60(0.99-2.60)$ & 0.058 \\
\hline Quartile $3(n=303)$ & $112(37.0)$ & $1.19(0.90-1.57)$ & 0.227 & $40(13.2)$ & $1.33(0.82-2.14)$ & 0.249 \\
\hline Quartile $4(n=290)$ & $99(34.1)$ & 1.00 (referent) & & $31(10.7)$ & 1.00 (referent) & \\
\hline
\end{tabular}

Abbreviations: $C V D$, cardiovascular disease; $B U A$, broadband ultrasound attenuation. Model 1: adjusted including age, BMI, and treatment (calcium versus placebo). Model 2: Model 1 plus smoking history, prevalent diabetes, CVD and cancer. Model 3: Model 2 plus total hip BMD in 1998/1999\# (assessed in 1130 participants). $P$ value represents from Cox proportional hazards regression with italicized Bonferroni-corrected $p$ values $\leq$ 0.017. $P$ for trend obtained using the median value of each quartile. The $p$ for trend for all-cause mortality was $\leq 0.001$ for models 1,2 , and 3. For CVD mortality, the $p$ for trend for CVD mortality was $0.013,0.019$, and 0.016 for models 1,2 , and 3 , respectively

\section{Discussion}

In this study, we identified a consistent and robust association between lower BUA and higher all-cause and CVD mortality over 15 years in older women. This relationship persisted after adjustment for a range of established cardiovascular risk factors as well as hip BMD. Importantly, calcaneal BUA remained associated with CVD mortality even after adjustments for estimated cardiovascular risk using conventional risk factors. This suggests that calcaneal BUA may capture additional prognostic information regarding future mortality risk in comparison to hip BMD.

For every SD reduction in calcaneal BUA, we observed a $15 \%$ and $20 \%$ increase in the multivariable-adjusted relative hazard for all-cause and CVD mortality, respectively. These findings are similar to those of Bauer et al. [18] who reported a $16 \%$ increase in the risk of all-cause and 19\% CVD mortality over 5 years ( $n=5816$ women, age $\sim 79$ years) per SD reduction in calcaneal BUA. Similarly, Browner et al. [33] measured calcaneal BMD by single-photon absorptiometry in a large cohort of elderly women ( $n=9704$, age $\geq 65$ years) and they reported only age adjusted $21 \%$ and $22 \%$ increase in the risk of non-trauma and cancer mortality, respectively. However, no relationship between calcaneal BMD and CVD mortality was observed (RR 1.12, $p=0.25$ ). A systematic review and meta-analysis of 11 studies reported that individuals with low BMD had a 33\% higher CVD risk [34]. Collectively, these findings suggest that measures of bone health including calcaneal BUA and BMD are related to all-cause and CVD mortality. Of importance, understanding how and why this relationship exists may shed light on novel aspects of bone and vascular aging.

Regarding potential mechanisms underlying these observations, there are numerous hypothesized mechanisms including shared genetic, dietary, and lifestyle factors [35]; loss of endogenous protective pathways [36]; and an overlap in physiopathological mechanisms between osteoporosis and calcified vasculature [37]. Increased release of reactive oxygen species and pro-inflammatory mediators and alteration of cell signaling related to bone cellular senescence that occur with aging may also explain the observed association between low BUA and all-cause and CVD mortality [37, 38]. Of interest, the persistence of the association between BUA and all-cause and CVD mortality after adjustment for hip BMD may be partly justified by the presence of additional genetic loci that are related to calcaneal BUA but not hip or spine BMD [39]. For example, TMEM135 (rs597319), a gene which is related to longevity, was identified as determinant of calcaneal bone properties, but not hip BMD [40].

The significant difference in the relationship between calcaneal BUA and all-cause and CVD mortality across different levels of circulating vascular inflammatory biomarkers 
Reduction in BUA, per SD $(8.0 \mathrm{db} / \mathrm{Mhz})$ stratified by tertiles of

All-cause mortality over 15 years

biomarkers

Lipocalin 2

T1: HR 1.32 95\%CI 1.11-1.56, p=0.001

T2: HR $1.1495 \%$ CI $0.96-1.37, \mathrm{p}=0.141$

T3: HR $0.9995 \%$ CI $0.86-1.14, \mathrm{p}=0.892$

Cystatin C

T1: HR $1.1895 \%$ CI $0.99-1.40), \mathrm{p}=0.074$

T2: HR $0.9895 \%$ CI $0.81-1.17, \mathrm{p}=0.788$

T3: HR $1.1195 \%$ CI $0.96-1.29, p=0.157$

Homocysteine

T1: HR 1.33 95\%CI 1.10-1.60, $\mathrm{p}=0.001$

T2: HR $1.0995 \%$ CI $0.93-1.27, \mathrm{p}=0.311$

T3: HR $1.0795 \%$ CI $0.91-1.26, \mathrm{p}=0.439$

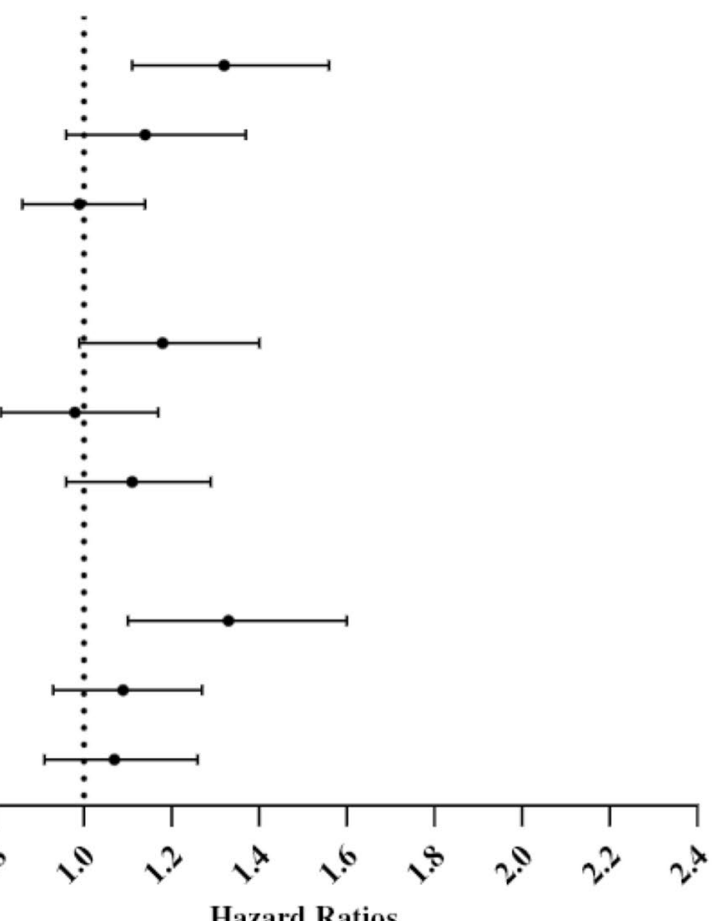

\section{CVD mortality over 15 years}

\section{Reduction in BUA, per SD $(8.0 \mathrm{db} / \mathrm{Mhz})$ stratified by tertiles of} biomarkers

\section{Lipocalin 2}

T1: HR $1.3695 \%$ CI 1.00-1.84, $\mathrm{p}=0.050$

T2: HR $1.2295 \%$ CI $0.92-1.61, p=0.170$

T3: HR $1.0595 \%$ CI 0.84-1.31, $\mathrm{p}=0.653$

\section{Cystatin C}

T1: HR $1.2995 \%$ CI $0.97-1.72, p=0.079$

T2: HR $1.1595 \%$ CI $0.84-1.56, \mathrm{p}=0.386$

T3: HR $1.0995 \%$ CI $0.87-1.36, p=0.459$

\section{Homocysteine}

T1: HR $1.6095 \% \mathrm{CI} 1.15-2.24, \mathrm{p}=0.006$

T2: HR $1.2495 \%$ CI $0.94-1.63, p=0.135$

T3: HR $0.9995 \% \mathrm{CI} 0.77-1.26, \mathrm{p}=0.921$

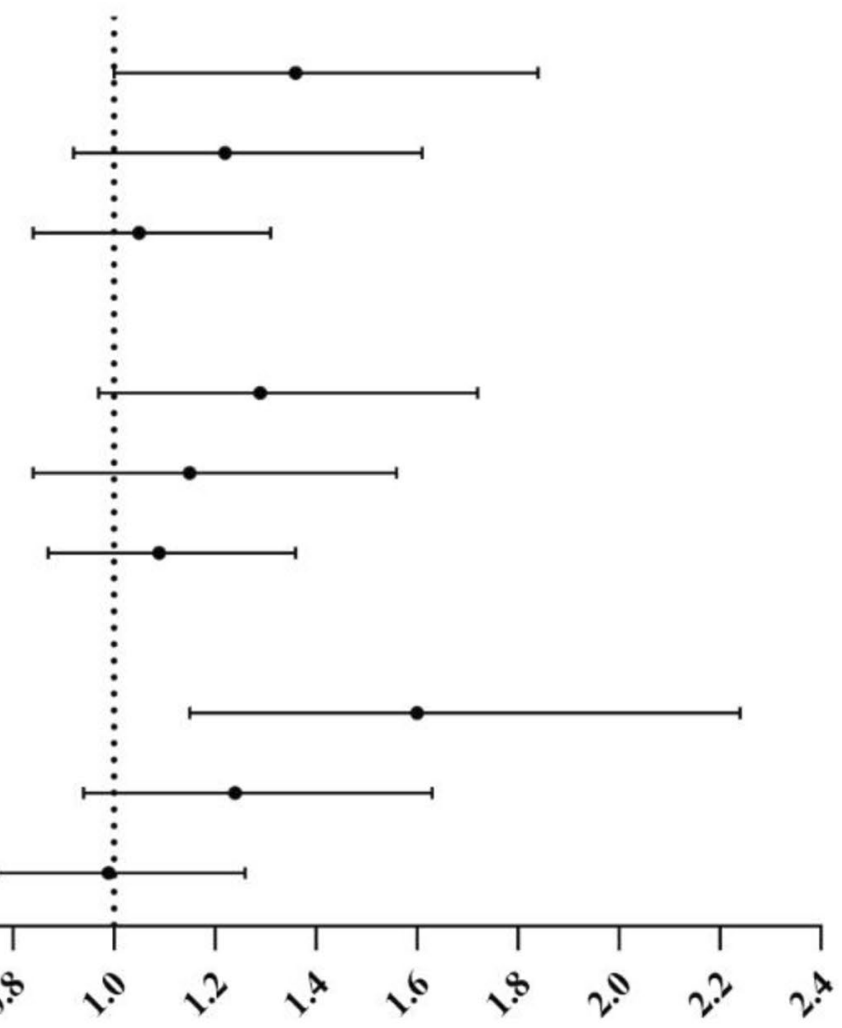

Hazard Ratios 
४Fig. 4 Adjusted hazard ratios per SD calcaneal broadband ultrasound attenuation (BUA) in relation to risk of $\mathbf{a}$ all-cause mortality and $\mathbf{b}$ CVD mortality. Categories for the tertiles of biomarkers included lipocalin 2 (T1<67.7, T2: 67.7 to $<87.9$, T3: $\geq 87.9 \mathrm{mg} / \mathrm{dL}$ ), cystatin $\mathrm{C}(\mathrm{T} 1:<0.97, \mathrm{~T} 2: 0.97$ to $<1.1, \mathrm{~T} 3: \geq 1.1)$, homocysteine $(\mathrm{T} 1:<9.9$, T2: 9.9 to $<12.7, \mathrm{~T} 3: \geq 12.7)$. The model is adjusted for age, BMI, and treatment code (calcium/placebo)

may be attributable to difference in cardiometabolic health of the cohorts. Specifically, we observed interactions between calcaneal BUA and inflammatory and/or metabolic biomarkers for all-cause and CVD mortality. The association between lower calcaneal BUA and all-cause mortality appeared stronger in the women with lowest circulating levels of lipocalin 2, cystatin C, and homocysteine. Given lower levels of these biomarkers are related to better cardiometabolic health outcomes, these findings suggest that age-related inflammatory and metabolic disorders may obscure the relationship between BUA and all-cause and CVD mortality. In addition to the inflammatory biomarkers, the presence of carotid plaques and a BMI $\leq 30 \mathrm{~kg} / \mathrm{m}^{2}$ was also found to potentially modify the association between BUA and all-cause mortality. Specifically, this relationship appeared stronger in non-obese women, and is supported by our analysis considering inflammatory biomarkers. Such findings may be attributed to the relationship between obesity, higher chronic inflammation, and metabolic dysfunction. Despite these novel findings, caution needs to be exercised when interpreting the results based on interaction testing. Such analyses are hypothesis-generating and should be considered tentatively until validated in other cohorts.

Lifestyle factors including diet, insufficient physical activity, and smoking are also known to negatively influence health outcomes, including obesity. Indeed, smoking and low physical activity were significantly associated with both calcaneal ultrasound measures and mortality outcomes in this study. Although nutritional factors were not examined here, its importance to musculoskeletal and vascular health is undeniable, warranting further investigation when examining the relationship between BUA, CVD, and mortality.

Despite these novel findings, limitations of this work must be acknowledged. Firstly, the observational nature of the study means that causality cannot be established. Nevertheless, to minimize residual confounding, we considered a detailed list of risk factors implicated in bone, cardiovascular, and general health. Secondly, hip BMD was obtained approximately 1 year after measurement of calcaneal ultrasound and was only available in $80 \%$ of the women. Thirdly, the study may not be applicable to younger population and older men. Finally, there was large unexplained variance ( 95\%) in our model when considering all-cause and CVD mortality in these older women. Nonetheless, the addition of BUA increased the amount of explained variance by $11.2 \%$ and $13.8 \%$ for all deaths and CVD deaths, respectively. However, strengths of this study include long-term follow-up (15 years) with asymptomatic measures of CVD, and death data obtained from linked heath records which are independent of self-report. This facilitates the accurate examination of the longitudinal relationship between baseline quantitative ultrasound measures and longterm clinical endpoints. We also considered a wide range of established CVD risk factors (e.g., inflammatory biomarkers, subclinical measures of CVD) often considered mechanistic when considering the relationship between bone, vasculature, and mortality outcomes [41]. Finally, extensive information regarding CVD risk factors, disease status, and lifestyle factors were considered in a cohort of older women representing a high-risk population for osteoporosis.

\section{Conclusion}

The findings of this study suggest that low calcaneal QUS, and in particular BUA, is associated with all-cause and CVD mortality in older women highlighting an association between bone and vascular health, which is an important consideration for healthy aging.

Supplementary Information The online version contains supplementary material available at https://doi.org/10.1007/s00198-022-06317-x.

Acknowledgements We would like to thank the staff at the Registry of Births, Deaths and Marriages and the Data Linkage Branch, Hospital Morbidity Data Collection, for their work to provide the data for this study.

Funding Open Access funding enabled and organized by CAUL and its Member Institutions. The study was supported by Kidney Health Australia grant S07 10, Healthway Health Promotion Foundation of Western Australia; Sir Charles Gairdner Hospital Research Advisory Committee Grant; and by project grants 254627, 303169, and 572604 from the National Health and Medical Research Council of Australia. The salary of JRL is supported by a National Heart Foundation of Australia Future Leader Fellowship (ID: 102817). The salary of MS is supported by a Royal Perth Hospital Research Foundation Career Advancement Fellowship (CAF 130/2020) and an Emerging Leader Fellowship from the Western Australian Future Health Research and Innovation Fund. DPK was supported by a grant from the National Institute of Arthritis, Musculoskeletal and Skin Diseases R01 AR041398. None of the funding agencies had any role in the conduct of the study; collection, management, analysis, or interpretation of the data; or preparation, review, or approval of the manuscript.

Data availability The data that support the findings of this study are available from the corresponding author upon reasonable request.

Code availability Not applicable.

\section{Declarations}

Ethics approval Ethical approval was obtained from the University of Western Australia Human Ethics committee (approval number 05/06/004/H50), while the data linkage study was approved by Human Research Ethics Committee of the Western Australian Department of Health (approval number \#2009/24). Written informed consent was obtained from all participants. 


\section{Conflict of interest None.}

Open Access This article is licensed under a Creative Commons Attribution-NonCommercial 4.0 International License, which permits any non-commercial use, sharing, adaptation, distribution and reproduction in any medium or format, as long as you give appropriate credit to the original author(s) and the source, provide a link to the Creative Commons licence, and indicate if changes were made. The images or other third party material in this article are included in the article's Creative Commons licence, unless indicated otherwise in a credit line to the material. If material is not included in the article's Creative Commons licence and your intended use is not permitted by statutory regulation or exceeds the permitted use, you will need to obtain permission directly from the copyright holder. To view a copy of this licence, visit http://creativecommons.org/licenses/by-nc/4.0/.

\section{References}

1. Warburton DE, Nicol CW, Gatto SN, Bredin SS (2007) Cardiovascular disease and osteoporosis: balancing risk management. Vasc Health Risk Manag 3:673-689

2. Khosla S, Farr JN, Tchkonia T, Kirkland JL (2020) The role of cellular senescence in ageing and endocrine disease. Nat Rev Endocrinol 16:263-275

3. Farhat GN, Cauley JA (2008) The link between osteoporosis and cardiovascular disease. Clin Cases Miner Bone Metab 5:19-34

4. Qu X, Huang X, Jin F, Wang H, Hao Y, Tang T, Dai K (2013) Bone mineral density and all-cause, cardiovascular and stroke mortality: a meta-analysis of prospective cohort studies. Int J Cardiol 166:385-393

5. Tran T, Bliuc D, Pham HM, van Geel T, Adachi JD, Berger C, van den Bergh J, Eisman JA, Geusens P, Goltzman D (2020) A risk assessment tool for predicting fragility fractures and mortality in the elderly. J Bone Miner Res 35:1923-1934

6. Szulc P, Kiel DP, Delmas PD (2008) Calcifications in the abdominal aorta predict fractures in men: MINOS study. J Bone Miner Res 23:95-102

7. Bagger Y, Tanko L, Alexandersen P, Qin G, Christiansen C, Group PERFS (2006) Radiographic measure of aorta calcification is a site-specific predictor of bone loss and fracture risk at the hip. J Intern Med 259:598-605

8. Lewis JR, Eggermont CJ, Schousboe JT, Lim WH, Wong G, Khoo B, Sim M, Yu M, Ueland T, Bollerslev J (2019) Association between abdominal aortic calcification, bone mineral density, and fracture in older women. J Bone Miner Res 34:2052-2060

9. Ueland T, Wilson SG, Amirul Islam FM, Mullin B, Devine A, Bollerslev J, Zhu K, Prince RL (2009) A cohort study of the effects of serum osteoprotegerin and osteoprotegerin gene polymorphisms on cardiovascular mortality in elderly women. Clin Endocrinol (Oxf) 71:828-833

10. Ueland T, Dahl CP, Kjekshus J et al (2011) Osteoprotegerin predicts progression of chronic heart failure: results from CORONA. Circ Heart Fail 4:145-152

11. Lim WH, Wong G, Lim EM, Byrnes E, Zhu K, Devine A, Pavlos NJ, Prince RL, Lewis JR (2015) Circulating lipocalin 2 levels predict fracture-related hospitalizations in elderly women: a prospective cohort study. J Bone Miner Res 30:2078-2085

12. Tanaka S, Ando K, Kobayashi K, Hida T, Ito K, Tsushima M, Morozumi M, Machino M, Ota K, Suzuki K (2019) Utility of the serum cystatin $\mathrm{C}$ level for diagnosis of osteoporosis among middle-aged and elderly people. BioMed Res Int 2019:1-6
13. Van Meurs JB, Dhonukshe-Rutten RA, Pluijm SM, van der Klift M, de Jonge R, Lindemans J, de Groot LC, Hofman A, Witteman JC, van Leeuwen JP (2004) Homocysteine levels and the risk of osteoporotic fracture. N Engl J Med 350:2033-2041

14. Raisi-Estabragh Z, Biasiolli L, Cooper J, Aung N, Fung K, Paiva JM, Sanghvi MM, Thomson RJ, Curtis E, Paccou J (2020) Poor bone quality is associated with greater arterial stiffness: insights from the UK Biobank. J Bone Mineral Res 36:90-99

15. Hans D, Dargent-Molina P, Schott A, Sebert J, Cormier C, Kotzki P, Delmas P, Pouilles J, Breart G, Meunier P (1996) Ultrasonographic heel measurements to predict hip fracture in elderly women: the EPIDOS prospective study. Lancet 348:511-514

16. Marín F, González-Macías J, Díez-Pérez A, Palma S, DelgadoRodríguez M (2006) Relationship between bone quantitative ultrasound and fractures: a meta-analysis. J Bone Miner Res 21:1126-1135

17. McCloskey EV, Kanis JA, Oden A, Harvey NC, Bauer D, González-Macias J, Hans D, Kaptoge S, Krieg MA, Kwok T (2015) Predictive ability of heel quantitative ultrasound for incident fractures: an individual-level meta-analysis. Osteoporos Int 26:1979-1987

18. Bauer D, Palermo L, Black D (2002) Quantitative ultrasound and mortality: a prospective study. Osteoporos Int 13:606-612

19. González-Macías J, Marin F, Vila J, Carrasco E, Benavides P, Castell M, Magaña J, Chavida F, Díez-Pérez A (2009) Relationship between bone quantitative ultrasound and mortality: a prospective study. Osteoporos Int 20:257-264

20. Prince RL, Devine A, Dhaliwal SS, Dick IM (2006) Effects of calcium supplementation on clinical fracture and bone structure: results of a 5-year, double-blind, placebo-controlled trial in elderly women. Arch Intern Med 166:869-875

21. Henzell S, Dhaliwal S, Pontifex R, Gill F, Price R, Retallack R, Prince R (2000) Precision error of fan-beam dual x-ray absorptiometry scans at spine, hip, and forearm. J Clin Densitom 3:359-364

22. Devine A, Dhaliwal SS, Dick IM, Bollerslev J, Prince RL (2004) Physical activity and calcium consumption are important determinants of lower limb bone mass in older women. J Bone Miner Res 19:1634-1639

23. Bruce DG, Devine A, Prince RL (2002) Recreational physical activity levels in healthy older women: the importance of fear of falling. J Am Geriatr Soc 50:84-89

24. McArdle WD, Katch FI, Katch VL (1991) Energy, nutrition and human performance. Lea \& Febiger, Philadelphia

25. Pollock ML (1978) Health and fitness through physical activity. John Wiley \& Sons Inc, New York, NY

26. D’Agostino RB Sr, Vasan RS, Pencina MJ, Wolf PA, Cobain M, Massaro JM, Kannel WB (2008) General cardiovascular risk profile for use in primary care: the Framingham Heart Study. Circulation 117:743-753

27. Lewis JR, Schousboe JT, Lim WH, Wong G, Wilson KE, Zhu K, Thompson PL, Kiel DP, Prince RL (2018) Long-term atherosclerotic vascular disease risk and prognosis in elderly women with abdominal aortic calcification on lateral spine images captured during bone density testing: a prospective study. J Bone Miner Res 33:1001-1010

28. Lewis JR, Zhu K, Thompson PL, Prince RL (2014) The effects of 3 years of calcium supplementation on common carotid artery intimal medial thickness and carotid atherosclerosis in older women: an ancillary study of the CAIFOS randomized controlled trial. J Bone Miner Res 29:534-541

29. Chong JJH, Prince RL, Thompson PL et al (2019) Association between plasma neutrophil gelatinase-associated lipocalin and cardiac disease hospitalizations and deaths in older women. J Am Heart Assoc 8:e011028 
30. World Health Organization (1977) Manual of the international statistical classification of diseases, injuries, and causes of death: based on the recommendations of the ninth revision conference, 1975, and adopted by the Twenty-ninth World Health Assembly. World Health Organization, Geneva

31. World Health Organization (2004) ICD-10: international statistical classification of diseases and related health problems: tenth revision. World Health Organization, Geneva

32. Harrell FE (2021) R Package 'rms': 1-254

33. Browner WS, Seeley DG, Vogt TM, Cummings SR (1991) Nontrauma mortality in elderly women with low bone mineral density. Lancet 338:355-358

34. Veronese N, Stubbs B, Crepaldi G, Solmi M, Cooper C, Harvey NC, Reginster JY, Rizzoli R, Civitelli R, Schofield P (2017) Relationship between low bone mineral density and fractures with incident cardiovascular disease: a systematic review and metaanalysis. J Bone Miner Res 32:1126-1135

35. Curtis GL, Chughtai M, Khlopas A, Newman JM, Khan R, Shaffiy S, Nadhim A, Bhave A, Mont MA (2017) Impact of physical activity in cardiovascular and musculoskeletal health: can motion be medicine? J Clin Med Res 9:375-381

36. Cannata-Andia JB, Roman-Garcia P, Hruska K (2011) The connections between vascular calcification and bone health. Oxford University Press 26:3429-3436
37. Stojanović SD, Fiedler J, Bauersachs J, Thum T, Sedding DG (2020) Senescence-induced inflammation: an important player and key therapeutic target in atherosclerosis. Eur Heart $\mathbf{J}$ 41:2983-2996

38. Kovacic JC, Moreno P, Nabel EG, Hachinski V, Fuster V (2011) Cellular senescence, vascular disease, and aging: part 2 of a 2-part review: clinical vascular disease in the elderly. Circulation 123:1900-1910

39. Mullin BH, Zhao JH, Brown SJ et al (2017) Genome-wide association study meta-analysis for quantitative ultrasound parameters of bone identifies five novel loci for broadband ultrasound attenuation. Hum Mol Genet 26:2791-2802

40. Moayyeri A, Hsu Y-H, Karasik D, Estrada K, Xiao S-M, Nielson C, Srikanth P, Giroux S, Wilson SG, Zheng H-F (2014) Genetic determinants of heel bone properties: genome-wide association meta-analysis and replication in the GEFOS/GENOMOS consortium. Hum Mol Genet 23:3054-3068

41. Ye C, Xu M, Wang S, Jiang S, Chen X, Zhou X, He R (2016) Decreased bone mineral density is an independent predictor for the development of atherosclerosis: a systematic review and metaanalysis. PLoS One 11:e0154740

Publisher's note Springer Nature remains neutral with regard to jurisdictional claims in published maps and institutional affiliations.

\section{Authors and Affiliations}

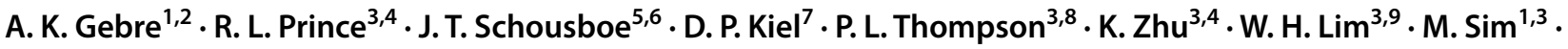 J. R. Lewis ${ }^{1,3,10}$}

1 Institute for Nutrition Research, School of Medical and Health Sciences, Edith Cowan University, Joondalup, WA 6027, Australia

2 School of Pharmacy, College of Health Sciences, Mekelle University, Mekelle, Ethiopia

3 Medical School, The University of Western Australia, Perth, Australia

4 Department of Endocrinology and Diabetes, Sir Charles Gairdner Hospital, Perth, Australia

5 Park Nicollet Osteoporosis Center and HealthPartners Institute, HealthPartners, Minneapolis, MN 55416, USA

6 Division of Health Policy and Management, University of Minnesota, Minneapolis, MN 55455, USA
7 Marcus Institute for Aging Research, Hebrew SeniorLife, Department of Medicine, Beth Israel Deaconess Medical Center and Harvard Medical School, Boston, MA 02215, USA

8 Department of Cardiology, Sir Charles Gairdner Hospital, Perth, Australia

9 Renal Department, Sir Charles Gairdner Hospital, Perth, Australia

10 Centre for Kidney Research, Children's Hospital at Westmead School of Public Health, Sydney Medical School, The University of Sydney, Sydney, Australia 